\title{
Regional longitudinal bi-ventricular function in pulmonary hypertension: single heart-beat assessment of strain by fast-senc imaging
}

Monda L Shehata ${ }^{1 *}$, Tamer A Basha', Ahmed A Harouni ${ }^{1}$, Jan Skrok', Sukhminder Singh ${ }^{1}$, Stephen Mathai ${ }^{1}$, Joao AC Lima', Nael F Osman', David A Bluemke², Paul M Hassoun', Jens Vogel-Claussen

From 2011 SCMR/Euro CMR Joint Scientific Sessions

Nice, France. 3-6 February 2011

\section{Purpose}

Right ventricular (RV) function is the most important determinant of survival in patients with pulmonary hypertension $(\mathrm{PH})$, thus monitoring of RV function is critical. However, RV regional function assessment is challenging using current MR tagging techniques. Thus, the purpose was to evaluate regional longitudinal ventricular deformation $\left(\mathrm{E}_{\mathrm{LL}}\right)$ acquired by free breathing single heart-beat fast strain encoded imaging (fast-SENC) in relation to global ventricular dysfunction markers and pulmonary hemodynamics in $\mathrm{PH}$ patients.

\section{Materials and methods}

48 subjects [35 $\mathrm{PH}$ patients (mean pulmonary arterial pressure $\mathrm{mPAP}=40.2 \pm 11.8 \mathrm{mmHg}$ ) and 13 age and gender matched controls] were examined using short axis fast-SENC MRI and cine imaging. All patients underwent right heart catheterization (RHC). Segmental (15 RV and $16 \mathrm{LV}$ segments), slice (basal, mid and apical), as well as mean (average of segments) peak systolic $\mathrm{E}_{\mathrm{LL}}$ were quantified for both ventricles and correlated with global function and RHC indices. Patients were stratified into 3 groups based on RV ejection fraction (RVEF).

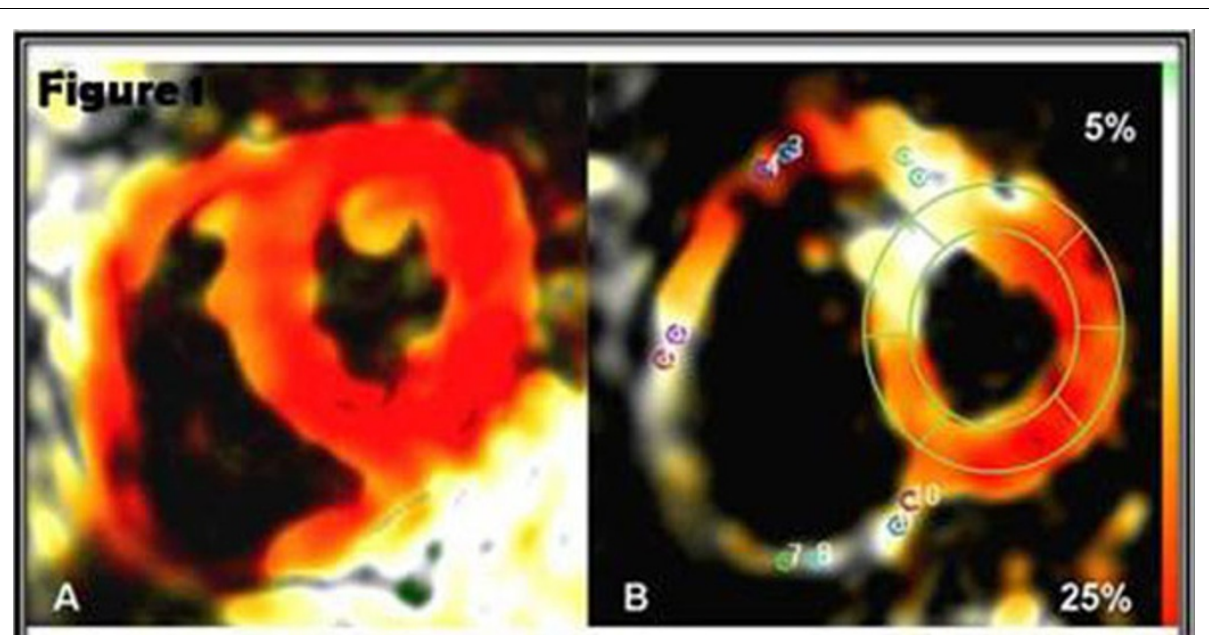

Figure 1 (A) Basal short axis fast-SENC peak systolic image in a 53 year old healthy female subject demonstrating normal longitudinal shortening $\left(E_{L L}\right)$ (red color). (B) Basal short axis fast-SENC peak systolic image in a 47 year old female PH patient ( $\mathrm{mPAP}=51 \mathrm{mmHg}$ ). Note regionally reduced RV $E_{L L}$ and $L V$ antero-septal $E_{L L}$ (white color). Probe points correspond to regions of interest where strain was measured. A mesh was used to measure LV ELL at six basal, six mid and four apical LV segments respectively.

'Johns Hokins University, Baltimore, MD, USA

Full list of author information is available at the end of the article

(c) 2011 Shehata et al; licensee BioMed Central Ltd. This is an open access article distributed under the terms of the Creative Commons Attribution License (http://creativecommons.org/licenses/by/2.0), which permits unrestricted use, distribution, and reproduction in any medium, provided the original work is properly cited. 
Table 1 Right ventricular longitudinal (ELL) strain in PH patients and controls

\begin{tabular}{llll}
\hline $\mathbf{E}_{\mathbf{L L}} \mathbf{( \% )}$ & $\mathbf{P H} \mathbf{2 5 \%}-\mathbf{7 5 \%} \mathbf{( N = \mathbf { 3 5 } )}$ & Control 25\% - 75\% (N =13) & P value \\
\hline Mean Ventricular Strain & $-19.3(-20.5--15.3)$ & $-21.3(-21.9--20.2)$ & 0.001 \\
Slices & $-19.1(-21.6--14.9)$ & $-21.7(-22.0--20.2)$ & 0.008 \\
Basal & $-19.3(-20.3--12.6)$ & $-21.8(-22.7--19.1)$ & 0.002 \\
Mid & $-18.1(-21.8--15.7)$ & $-20.9(-22.3--19.8)$ & 0.02 \\
Apical & & & $<0.001$ \\
Segments & $-14.1(-18.8--12.5)$ & $-20.2(-23.1--17.1)$ & 0.02 \\
Basal Anterior Septal Insertion & $-21.2(-24.1--15.1)$ & $-24.0(-25.1--21.8)$ & 0.03 \\
Basal Anterior & $-21.0(-25.1--17.9)$ & $-23.8(-26.2--22.5)$ & 0.09 \\
Basal Lateral & $-18.3(-21.6--15.1)$ & $-20.2(-23.6--18.6)$ & 0.88 \\
Basal Inferior & $-19.9(-21.6--14.1)$ & $-19.1(-21.7--16.3)$ & $<0.001$ \\
Basal Inferior Septal Insertion & $-15.1(-18.1--9.6)$ & $-20.8(-22.7--17.7)$ & $<0.001$ \\
Mid Anterior Septal Insertion & $-16.3(-19.1--12.5)$ & $-22.1(-25.1--21.3)$ & 0.005 \\
Mid Anterior & $-22.5(-23.6--15.0)$ & $-24.9(-25.9--22.9)$ & 0.16 \\
Mid Lateral & $-20.1(-23.3--14.1)$ & $-20.1(-24.6--18.7)$ & 0.49 \\
Mid Inferior & $-16.9(-21.2--11.1)$ & $-18.7(-20.1--16.1)$ & 0.02 \\
Mid Inferior Septal Insetion & $-16.6(-20.7--12.3)$ & $-20.4(-22.3--19.0)$ & 0.01 \\
Apical Anterior Septal Insertion & $-18.4(-21.2--14.9)$ & $-21.9(-23.8--18.8)$ & 0.0009 \\
Apical Anterior & $-20.4(-24.4--15.4)$ & $-24.1(-25.7--22.1)$ & 0.08 \\
Apical Lateral & $-18.3(-24.1--13.1)$ & $-22.2(-24.6--19.7)$ & 0.84 \\
Apical Inferior & $-18.1(-21.8--12.8)$ & $-17.3(-19.5--15.6)$ & \\
Apical Inferior Septal Insertion & & \\
\hline
\end{tabular}
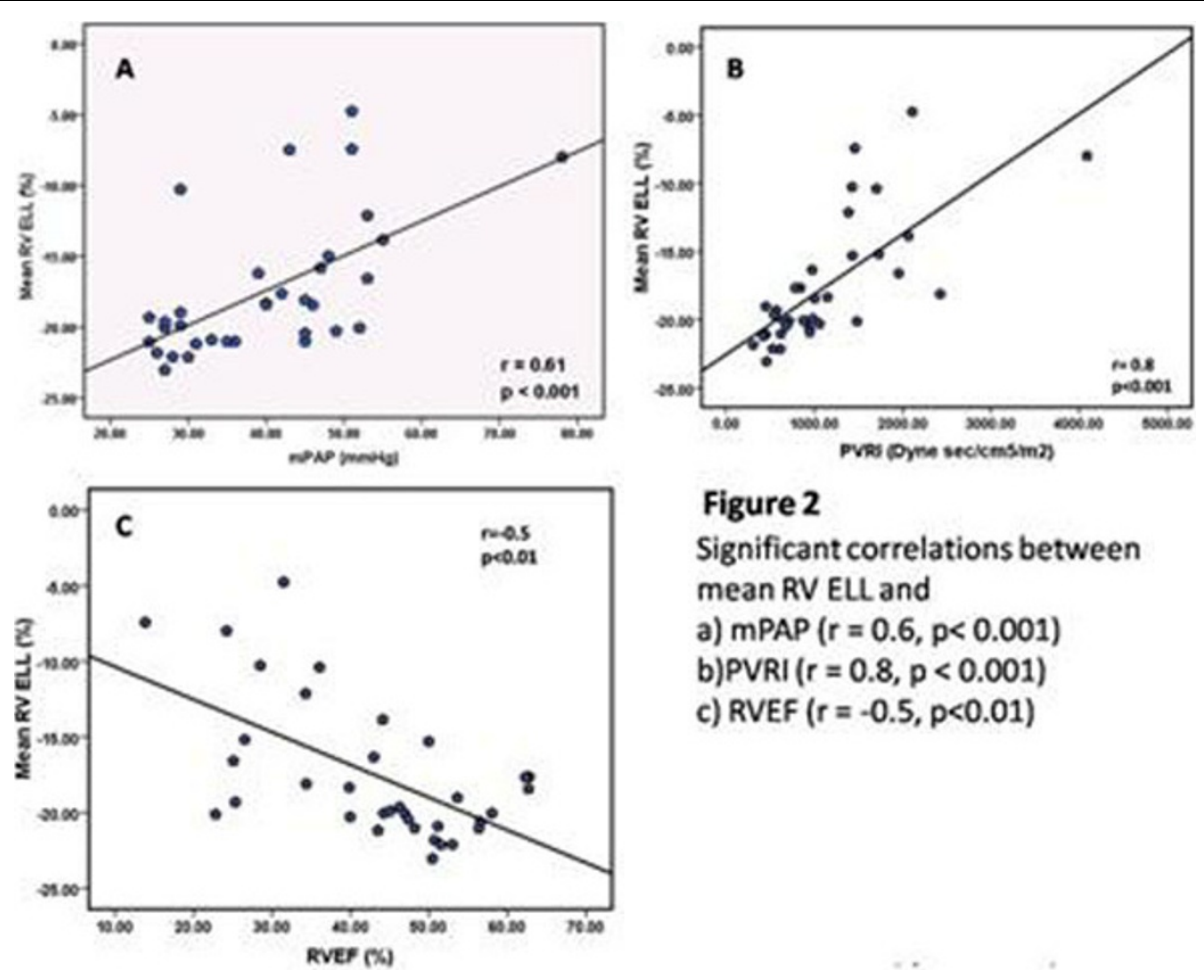

Figure 2

Significant correlations between mean RV ELL and

a) $\operatorname{mPAP}(r=0.6, p<0.001)$

b) PVRI $(r=0.8, p<0.001)$

c) $\operatorname{RVEF}(r=-0.5, p<0.01)$

Figure 2 


\section{Results}

PH patients demonstrated reduced $E_{L L}$ at all RV levels compared to controls (Figure 1). On regional analysis, reduced $E_{L L}$ was noted in all segments $(p<0.05)$ except the inferior and inferior septal insertion at all levels (Table 1). Reduced mean RV $\mathrm{E}_{\mathrm{LL}}$ correlated with elevated $\mathrm{mPAP}(\mathrm{r}=0.6, \mathrm{p}<0.001)$, pulmonary vascular resistance index (PVRI, $r=0.8, \mathrm{p}<0.001$ ) as well as reduced $\mathrm{RV}$ systolic function parameters ( $\mathrm{p}<0.05$ for all) (Figure $2)$. In the $L V$, reduced $E_{L L}$ was mainly noted at the basal anterior (-16.7 vs. $-20.5, \mathrm{p}=0.03)$ and antero-septal regions $(-12.8$ vs. $-18.2, \mathrm{p}<0.01)$. Reduced LV anteroseptal $E_{L L}$ correlated with increased mPAP $(r=0.5$, $p<0.01)$, increased septal eccentricity index $(r=0.5$, $\mathrm{p}<0.01)$ and reduced RV systolic function $(\mathrm{p}<0.05$ for all). On multiple linear regression including mPAP, RV end-diastolic volume index and RV mass index as covariates, mPAP was an independent predictor of reduced mean $R V E_{L L}(\beta=0.19, p<0.01)$. In turn, reduced mean $R V E_{L L}$ was the main predictor of reduced RVEF in $P H$ patients $(\beta=-1.2, \mathrm{p}=0.03)$ in a model including mPAP, mean RV $E_{L L}$ and RV mass index. In $\mathrm{PH}$ patients with maintained global $R V$ function, regional $E_{L L}$ was reduced at the basal and mid anterior septal insertions as well as mid anterior RV segments ( $\mathrm{p}<0.05$ for all).

\section{Conclusion}

In $\mathrm{PH}$ patients, reduced RV $\mathrm{E}_{\mathrm{LL}}$ measured by fast-SENC is associated with increased afterload and correlates with biventricular global dysfunction. RV strain analysis using fast-SENC can detect subclinical regional dysfunction in absence of global RV functional compromise.

\section{Author details}

${ }^{1}$ Johns Hokins University, Baltimore, MD, USA. ${ }^{2}$ National Institutes of Health, Bethesda, MD, USA.

Published: 2 February 2011
doi:10.1186/1532-429X-13-S1-P279

Cite this article as: Shehata et al:: Regional longitudinal bi-ventricular function in pulmonary hypertension: single heart-beat assessment of strain by fast-senc imaging. Journal of Cardiovascular Magnetic Resonance 2011 13(Suppl 1):P279.

\section{Submit your next manuscript to BioMed Central} and take full advantage of:

- Convenient online submission

- Thorough peer review

- No space constraints or color figure charges

- Immediate publication on acceptance

- Inclusion in PubMed, CAS, Scopus and Google Scholar

- Research which is freely available for redistribution

Submit your manuscript at www.biomedcentral.com/submit 